\title{
Ontological approach to modelling of discrete event dynamic system
}

\author{
V. KULBA ${ }^{1 *}$, S. NIKOLSKY ${ }^{2}$, and O. ZAIKIN ${ }^{3}$ \\ ${ }^{1}$ Institute of Control Sciences, Russian Academy of Science, 65 Profsoyuznaya St., 117997 Moscow, Russia \\ ${ }^{2}$ Moscow State Institute of Electronics and Mathematics, 3/12 Bolshoy Vuzovsky St., 109028 Moscow, Russia \\ ${ }^{3}$ Szczecin University of Technology, 48 Piastów, 70-311 Szczecin, Poland
}

\begin{abstract}
The mathematical model of discrete event dynamic system is considered. The main problem was to construct this model in Event-space so that it should be conceptually based on the same idea that this of differential system models generally given in the algebraic form as an action of monoïd in State-space. The construction was realized by using the Ontological approach.
\end{abstract}

Key words: discrete event dynamic system, ontological approach, metaontology.

\section{Introduction}

Conceptually the notion of discrete event dynamic system (DEDS) means a point of view on the nature of processes or dynamic systems which is needed in various domains of practical applications. In very general terms such a system is the object of researches that changes its states in the function of events that happen inside system itself. Now there is a variety of DEDS models in the form of automata, Petri nets, process algebra, temporal Petri nets, $(\max ,+)$-algebra and etc. Often these models were opposed to differential dynamic system models. But it is still actually to join these two points of view on mathematical dynamic system models, see for example [1]. The purpose of this paper is to construct mathematical DEDS models so that they should be conceptually common to the differential system models.

In this paper we focus on ontological approach [2, 3] to the synthesis of mathematical discrete event dynamic system models by using any ontology or Conceptual Semantic System [3].

This paper is organized as follows. Section 2 presents the ontological approach and defines General Problem of Realization (GRP). As a general example it presents the GPRsolutions for the natural metaontology. Section 3 contains the dynamic system mathematical models specifically chosen for the purpose of this paper. Section 4 presents metaontology "physical point". Section 5 presents the DEDS mathematical models that were obtained as the GRP-solutions for metaontology "physical point".

\section{Ontological approach}

The importance of ontology for investigation of the nature of modeling process has arisen in modern information modeling system theory [4]. Ontology fixes the principle that the information modeling system or modeling process (MP) begins on early stages of software life cycle where it has the form of conceptual analysis [3, 4].
From general point of view any ontology Ont is a totality of concepts

$$
\text { Ont }=\left(\mathrm{c}_{1}, \mathrm{c}_{2}, \ldots, \mathrm{c}_{\mathrm{N}}\right) .
$$

If the concepts $\left(c_{1}, c_{2}, \ldots, c_{N}\right)$ are of high logical level the ontology Ont is called high level ontology or metaontology MetaOnt [4]. It's to be noted that ontology Ont may contain only one concept, for example, object.

Ontology Ont being considered as a vocabulary generates its own modeling language $£(O n t)$ that may be given in linguistic or logic form. Thus it may be said that $£(O n t)$ represents the $£$-form of information modeling system MP. In this sense MetaOnt generates the modeling language of high logical level corresponding to the modeling in conceptually general case.

2.1. Concept diagram. Any ontology Ont may be considered as a conceptual form or $C$-form of information system MP. Modeling in C-form means the description of any "object of researches" in terms of concepts from ontology Ont.

Let $\mathrm{H}$ be "object of researches". The following diagram of characterization of "object of researches" $\mathrm{H}$

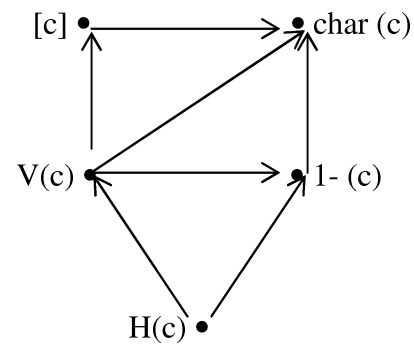

is called diagram of concept $\mathrm{C}$ or [c]-diagram [2]. Its elements

$$
<[\mathrm{c}], \mathrm{V}(\mathrm{c}), \operatorname{char}(\mathrm{c}), 1-(\mathrm{c})>
$$

are the characters of $\mathrm{H}(\mathrm{c})$ :

- character [c] is the "naming form" (name) of concept C;

- character V(c) is the "volume" of concept C;

*e-mail: Kulba@ipu.rssi.ru 
- character char (c) is the "attributes" of elements of V(c);

- character 1- (c) is "one element of volume of concept C".

In the logical sense the characters from (1) are related to $\mathrm{H}$ and thus they can be considered as the elementary conceptual models of "object of research" $\mathrm{H}$ when it is related to the volume of concept " $\mathrm{C}$ " what is noted by $\mathrm{H}(\mathrm{c})$ in [c]-diagram.

Thus [c]-diagram defines conceptual modeling as the characterization of "object of research" $\mathrm{H}$ in terms of elementary characters or conceptual models from (1), corresponding to the concepts from Ont.

The following party of [c]-diagram

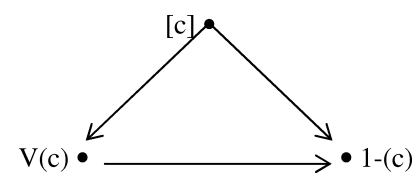

indicates the characters $\mathrm{V}(\mathrm{c})$ and 1-(c) as the meanings of naming form [c] and so it shows that they define two universal levels of conceptual semantic modeling, namely,

- V-modeling level - the modeling of $\mathrm{H}$ in terms of "volume";

- 1-modeling level - the modeling of $\mathrm{H}$ in terms of "one element of volume".

The arrow

$$
[\mathrm{c}] \Rightarrow 1-(\mathrm{c}) \text {, }
$$

in the last diagram is called General Problem of Realization [2]. This Problem distinguishes the 1-modeling level as essential. If in general terms the totality is supposed to be the volume $\mathrm{V}(\mathrm{c})$ of any concept $\mathrm{C}$ then to solve this problem means to construct the model of "one element of totality" which is initially assumed to be the "point" or "nonstructural whole".

The Ontological approach formulated in the form of the General Problem of Realization is the logically correct approach to the mathematical structures construction that joins conceptual and mathematical levels of information modeling system MP [2].

2.2. Natural metaontology. The main question of Ontological approach is the question if and how the metaontology being assumed as any vocabulary related to the £-form of information system MP leads to the mathematical modeling structures.

The [c]-diagram allows answer it by analysis of Solution of General Problem of Realization (or GRP-solutions) for the metaontology

$$
\text { MetaOnt }{ }^{\mathrm{NAT}}=(\text { object }, \text { propriety, relation, operation })
$$

This metsontology may be called natural because its concepts correspond to the subject-predicate structure of sentences of natural language that is the first $£$-form of information modeling system MP.

The analysis of [c]-diagram for each of elements of MetaOnt $^{\text {NAT }}$ shows that in assumption

$$
\text { [totality] is a [set }] \text { is a [object }]
$$

all the concepts from MetaOnt ${ }^{\mathrm{NAT}}$ are reduced to only concept [2]

\section{[object]}

Thus MetaOnt ${ }^{\mathrm{NAT}}$ is conceptually reduced to the metaontology of only one concept

$$
\mathrm{MetaOnt}^{\mathrm{Ob}}=\text { (object). }
$$

and General Problem of Realization has the form of the transformation-arrow

$$
\text { [ob] } \Rightarrow 1-(\mathrm{ob})
$$

It was also shown [2] that in assumption

$$
\text { [totality] is a [category] }
$$

the character $1-(\mathrm{ob})$ presents the totality whose elements are the following mathematical structures:

$$
\left\{\begin{array}{l}
<\mathrm{A}>- \text { set } \\
<\mathrm{AR}>- \text { relation system } \\
<\mathrm{AF}>- \text { algebra }
\end{array}\right.
$$

Thus object has three forms

$$
1-(\mathrm{ob}) \Rightarrow(<\mathrm{A}>,<\mathrm{AR}>,<\mathrm{AF}>),
$$

where set A is its Support and its Signature is composed from relations $\mathrm{R}$ and operations $\mathrm{F}$. It is necessary to notice that the elements of Signature $\mathrm{R}$ and $\mathrm{F}$ have the representations in the elements of set-support A in the form of tables or graphs.

In Logic, each element of Support A is called "elementary object" then on 1-modeling level each of structures (3) may be called "complex object".

Thus the GPR-solutions for MetaOnt ${ }^{\text {NAT }}$ verify the following diagram

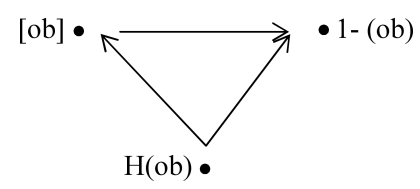

corresponding to structural operation

$$
\text { str: } \mathrm{H}(\mathrm{ob}) \bullet \Rightarrow 1-(\mathrm{ob})
$$

where object $\mathrm{H}(\mathrm{ob}) \bullet$ is a "point" or "nonstructural whole".

In accordance with this diagram each mathematical structure from (3) is the particular GRP-solution which generates its own modeling language $£\left(\right.$ MetaOnt $\left.{ }^{\mathrm{NAT}}\right)$ explaining "object of research" $\mathrm{H}$ in terms of concept "complex object".

The last diagram also shows that on the 1-modeling level for MetaOnt ${ }^{\text {NAT }}$ each element of Support of any "complex object" turns to be "elementary class"

$$
\mathrm{ob}=<[\mathrm{ob}], \mathrm{A}>, \mathrm{A} \subseteq \mathrm{V}(\mathrm{ob})
$$

or "elementary conceptual object".

Thus the analysis of General Problem of Realization for metaontology MetaOnt ${ }^{\text {NAT }}$ shows that the common form of all its Solutions, i.e. "complex objects" from 1-(ob) (3) is a pair

$$
\mathrm{ST}(o b)=<\text { Support, Signature }>,
$$


which can be called object structural type noted here by $\mathrm{ST}(o b)$.

So, for any concept $\mathrm{C}$ the Ontological approach leads to the General Problem of Realization. The complex GPRsolutions are the structural types ST(c) representing the models of "one element of volume V(c)". Thus it leads to the choice of metaontology that conceptually defines the elements of Support of ST(c) and it may be more complex than MetaOnt ${ }^{O b}$ that contains only one concept "object".

The natural power of MetaOnt ${ }^{\mathrm{NAT}}$ is that the concepts relation and operation corresponding to Signature elements of ST $(o b)$ may be used in modeling structures of any other metaontology with any other naming form of concepts that define the elements of its structural types Support.

\section{Metaontology "dynamic system"}

Dynamic system is the concept of high logical level. So it defines

$$
\text { MetaOnt }^{\mathrm{DS}}=(\text { dynamic system })=(\mathrm{DS}) .
$$

There are the following well-known mathematical models of 1-(DS) $[5,6]$ :

a) Geometrical model $D S$ : Vector field in the State space $\mathrm{S}$ of a system

$$
V: \mathrm{s} \rightarrow v(\mathrm{~s}),
$$

where $\mathrm{s} \in \mathrm{S}, v(\mathrm{~s})$ - vector outgoing from point $\mathrm{s}-$ state of a system.

b) Temporal model $D S$ :

$$
\text { әT: } \mathrm{T} \rightarrow \mathrm{S} \text { - temporal dynamics, }
$$

where $\mathrm{T}-$ set of time elements, $\mathrm{S}$ is a State space of a system.

c) State model DS:

$$
\partial \mathrm{S}: \mathrm{S} \rightarrow \mathrm{S}-\text { state dynamics, }
$$

where S - state space of system.

d) Net model DS:

$$
\mathrm{N}(\mathrm{DS})=<\mathrm{S}, \mathrm{P}, \rightarrow, \mathrm{s}_{0}>,
$$

where $\mathrm{S}-$ set of states, $\mathrm{P}-$ set of actions names; $\rightarrow \subseteq$ $\mathrm{S} \times \mathrm{T} \times \mathrm{S}-$ transfer relation in $\mathrm{S} ; \mathrm{s}_{0} \in \mathrm{S}$ - initial state.

Each transfer $\left(\mathrm{s}, \mathrm{p}, \mathrm{s}^{\prime}\right) \in \mathrm{S} \times \mathrm{T} \times \mathrm{S}$ corresponds to the assertion

$\mathrm{p}\left(\mathrm{s}, \mathrm{t}, \mathrm{s}^{\prime}\right)=\ll$ "action with the name $[p]$ moves the state $\mathrm{s}$ into the state $\mathrm{s}^{\prime} \gg$.

e) Algebraic model DS:

$$
\operatorname{Alg}(\mathrm{DS})=\{\mathrm{S}, \mathrm{M}, \mathrm{Y}, \delta, \beta\}
$$

where $\mathrm{M}$ - monoïde, $\mathrm{S}$ - state space, $\mathrm{Y}$ - output space, $\delta$ - system dynamics, $\beta: S \rightarrow$ Y output mapping. System dynamic $\delta$ is the action of monoide $\mathrm{M}$ in $\mathrm{S}$

$$
\delta: \mathrm{S} \times \mathrm{M} \rightarrow \mathrm{S}
$$

The dynamic system models mentioned above are the 1(DS) models and they may be considered as a GPR-solution in MetaOnt ${ }^{\mathrm{DS}}$. Their common form is structural type

$$
\mathrm{ST}(\text { state })=<\mathrm{S}, \mathrm{R}, \mathrm{F}>\text {. }
$$

The state space $\mathrm{S}$ is its Support whose elements conceptually related to the concepts "state" as the "state itself" or the "state of the system". Their Signature is conceptually related to the concepts "relation" $\mathrm{R}$ and "external operation" $\mathrm{F}$ defined in the State space. The basic geometrical idea of these models is in the following conceptual equivalence: "DS is a totality of directions in the state space S".

\section{Metaontology "discret event dynamic system"}

Taking in account the purpose of this paper let's now construct the GPR-solutions for metaontology

$$
\text { MetaOnt }{ }^{\text {DEDS }}=(\text { discrete event dynamic system }) \text {. }
$$

As it derives from Sec. 3 the modeling language $£(D E D S)$ includes the naming forms of the concepts "state" and "event". So initially any DEDS may be conceptually determined in terms of natural structural types (3), for example

$$
\Sigma=<\mathrm{E}^{*}, \mathrm{R}, \mathrm{F}>, \mathrm{E}^{*} \subset \mathrm{E} .
$$

Depending on the choice between the two mentioned concepts the totality $\mathrm{E}$ is the set of states or the set of events provided with suitable relations $\mathrm{R}$ and operations $\mathrm{F}$.

Besides in accordance with structural principle (4) any DEDS may be considered to be elementary or complex. The complex DEDS has the form of

$$
\Sigma=<\Sigma ; \mathrm{R}\left(\sigma_{\mathrm{k}}, \sigma_{\mathrm{j}}\right)>,
$$

where the relation $\mathrm{R}\left(\sigma_{\mathrm{k}}, \sigma_{\mathrm{j}}\right)$ is defined on the set of elementary DEDS

$$
\Sigma=\left\{\sigma_{\mathrm{j}} \mid \sigma_{\mathrm{j}}-\text { elementary, } \mathrm{i}=1, \mathrm{n}\right\} .
$$

So any DEDS is discrete in structural sense.

4.1. Metaontology "physical point". The structures types from (6) are the GPR-solutions for MetaOnt ${ }^{\text {DEDS }}$ having the Signature of natural structural types (3) in space $E^{*} \subset \mathrm{E}$ and they are evidently the object-oriented in this sense. But their Support is conceptually determined in the terms "state" and "event". So GPR-solutions for MetaOnt ${ }^{\text {DEDS }}$ are (state or event)-oriented in this sense. Thus it should be more logically correct to construct their Support so that it should be conceptually based on the concept "object" also.

In Logic any ontology Ont determines the assumptions about the types of entities that exist in opinion of any researcher. As it was marked in Physics "the physical point exists because of events that happen in it".

Let's consider the metaontology

$$
\text { MetaOnt }^{\mathrm{EVT}}=\{\text { object, state, time }\}
$$

supplemented with the following logical diagram LD 


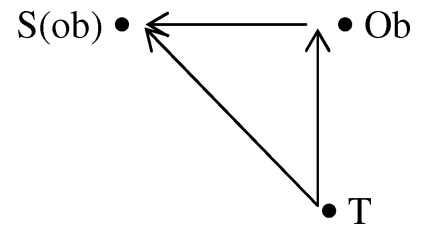

where $\mathrm{Ob}$ - totality of objects, S(ob) - totality of states of objects, $\mathrm{T}$ - totality of elements of time.

The logical links marked in diagram LD by correspondence-arrows distinguish the Event space:

$$
\text { Evt }=\{\text { evt } \mid \text { evt }=(\mathrm{ob}, \mathrm{s}, \mathrm{t})\}
$$

where $\mathrm{ob} \in \mathrm{Ob}, \mathrm{s} \in \mathrm{S}(\mathrm{ob}), \mathrm{t} \in \mathrm{T}$.

Each evt $=(\mathrm{ob}, \mathrm{s}, \mathrm{t}) \in$ Evt called "physical point" (nonstructural dynamic whole) determines two dynamic types linked with any object ob $\in \mathrm{Ob}$ :

- event with the object:

$$
\operatorname{evt}(\mathrm{ob})=(o b, s)
$$

due to $(\mathrm{Ob}, \mathrm{S}(\mathrm{ob}))$-arrow in $\mathrm{LD}$;

- element of object dynamics:

$$
\operatorname{evt}(s)=(s, t),
$$

due to $(\mathrm{T}, \mathrm{S}(\mathrm{ob}))$-arrow in LD.

So metaontology MetaOnt ${ }^{\text {Evt }}$ may be called "metaontology of physical point". This metaontology generates modeling language $£\left(\right.$ MetaOnt $\left.^{\text {Evt }}\right)$ which explains each "object of research" $\mathrm{H}$ in terms of "physical point" or triplet (ob, $s, t)-$ "event with the object ob on the element $(s, t)$ of its dynamics".

4.2. Structural system types of MetaOnt ${ }^{\text {Evt }}$. Metaontology MetaOnt ${ }^{\text {Evt }}$ joins the concepts "state" and "event" from MetaOnt ${ }^{\text {DEDS }}$ with the concept "object" from MetaOnt ${ }^{\text {NAT }}$. That allows the logically correct construction of eventoriented GPR-solutions in MetaOnt ${ }^{\text {DEDS }}$ in the form the extension of object-oriented GPR-solutions of MetaOnt ${ }^{\mathrm{NAT}}$ to the event space on the base of structural principle (4) and (8). In accordance with structural principle the event-oriented GPRsolutions for MetaOnt ${ }^{\text {DEDS }}$ are the structural types ST(event) whose Support is event space Evt. Besides the elements of Evt are the "elementary conceptual objects"

$$
\mathrm{ob}=<[\mathrm{ob}], \mathrm{A}>, \mathrm{A} \subseteq \mathrm{V}(\mathrm{ob}) .
$$

Thus in accordance with the purpose of this paper it's necessary to choice the suitable structural type ST(ob) so that ST(event) should be constructed as its extension to Evt

$$
\text { Ext: ST(ob) } \Rightarrow \text { ST(event). }
$$

The general idea of GPR-solutions for MetaOnt ${ }^{\mathrm{DS}}$ as well as for differential equations consists in conceptual equivalence: "DS is a totality of directions or vector field in the state space S". The results of analysis of DS models in Sec. 3 show that the suitable ST(ob) is monoide $M$ whose action $\sigma(5)$ in state space $S$ has the form of the set of elementary correspondences in $\mathrm{S}$ :

$$
\sigma^{\rightarrow}=\left\{|\rightarrow|(s, m) \mid \rightarrow s^{\prime}\right\}
$$

where $\mathrm{s}, \mathrm{s}^{\prime} \in \mathrm{S}, \mathrm{m} \in \mathrm{M}$.
The correspondence is the basic concept for definitions of such elements of ST(ob) structural types of MetaOnt ${ }^{\mathrm{NAT}}$ as relation and operation [7].

So conceptually the metaontology MetaOnt ${ }^{\mathrm{NAT}}$ is reduced to metaontology of only two concepts object and correspondence or in symbolic form $<\mathrm{ob}, \rightarrow>$.

The importance of correspondence as a basic element of the logical structure of mathematical modeling languages is the general idea of categorial form of MetaOnt ${ }^{\mathrm{NAT}}$ mathematics. On the other hand, correspondence is also important for the DS models because it turns to be the direct conceptual analogue of direction or element of vector field.

So, the event-oriented GPR-solutions ST(event) for MetaOnt ${ }^{\text {DEDS }}$ may be constructed on the base of categorial form of structural types $\mathrm{ST}(\mathrm{ob})=\mathrm{M}$, MetaOnt ${ }^{\mathrm{Ev}}$.

Monoide $\mathrm{M}$ being the category [7], each element $\mathrm{m} \in$ $M$ coincides with the correspondence-arrow $\mid \rightarrow$ from $\sigma^{\rightarrow}$. Then each operation from Signature of ST(event) type may be given as the set $\mathrm{A}(\rightarrow)$ of the elementary operational correspondence-arrows

$$
1-(\rightarrow)=\left\langle\left(\mathrm{s}^{\rightarrow}\right) \text {; dom }\left[{ }^{\mathrm{cen}}[\mid \rightarrow]\right] \operatorname{cod}\right\rangle
$$

where symbol $[\mid \rightarrow]-$ arrow's name, symbol s $\rightarrow-$ arrow's indicator and symbols dom - start, cen - center and cod end are the categorial variables associated with the arrow.

Thus the operation Ext (8) is the extension of categorial representation of monoid action in Evt-space.

\section{DEDS mathematical models}

The common mathematical model of DEDS in Evt is the ordered set

$$
\mathrm{ST}(\text { event })=<\text { Evt, } \leq>.
$$

In accordance with structural principle (4), (8) it supposes two essential types of Elementary DEDS. The first due to (T, S(ob))-arrow of LD-diagram is the Elementary temporal DEDS

$$
\sigma^{\mathrm{T}}=<\mathrm{ob}, \partial(\mathrm{ob})>,
$$

where $\partial(\mathrm{ob}): \mathrm{T} \rightarrow \mathrm{S}(\mathrm{ob})$ is the dynamics of object ob and the other is the Elementary Operational DEDS due to $(\mathrm{Ob}$, $\mathrm{S}(\mathrm{ob})$ )-arrow of this diagram.

\subsection{The Categorial model of Elementary Operational} DEDS. The abstract category is a mathematical structure composed from Objects, Arrows and Operation noted by Int that attributes the Arrows to the Objects.

Let Arrows be the elements of set $\mathrm{A}(\rightarrow)$ and Objects be the elements of Evt. Then Operation Int consists in representation of arrow 1- $\rightarrow)$ in Evt that is given as follows:

$$
\left\{\begin{array}{c}
\text { dom } \Rightarrow \mathrm{evt}_{0}=\left(\mathrm{ob}_{0}, \mathrm{~s}_{0}, \mathrm{t}_{0}\right) \\
\text { cen } \Rightarrow \mathrm{evt}_{1}=\left(\mathrm{ob}_{1}, \mathrm{~s}_{1}, \mathrm{t}_{1}\right) \\
\operatorname{cod} \Rightarrow \mathrm{evt}_{2}=\left(\mathrm{ob}_{2}, \mathrm{~s}_{2}, \mathrm{t}_{2}\right) \\
{[\mid \rightarrow] \Rightarrow \tau(\mid \rightarrow)}
\end{array}\right.
$$


the time interval $\tau(\rightarrow)$ is the temporal meaning attributed to the arrow $[\mid \rightarrow]$.

Logically the operation $\operatorname{Int}[1-(\rightarrow)]$ is the following correspondence

$$
\text { Int: } 1-(\rightarrow) \Rightarrow \operatorname{evt}_{0}\left[{ }^{\text {evt1 }}[\mid \rightarrow]\right] \mathrm{evt}_{2} \text {, }
$$

where $\mathrm{evt}_{0}, \mathrm{evt}_{1}, \mathrm{evt}_{2} \in$ Evt. The result of $\operatorname{Int}[1-(\rightarrow)]$

$$
\sigma^{\mathrm{F}}: \mathrm{evt}_{0}\left[{ }^{\mathrm{evt} 1}[\mid \rightarrow]\right] \mathrm{evt}_{2}
$$

is called Elementary Operational DEDS in Event-space Evt.

The totality of states of objects $\mathrm{S}(\mathrm{ob})$ is given as follows

$$
\mathrm{S}(\mathrm{ob})=<\mathrm{S}_{1}(\mathrm{ob}), \mathrm{S}_{2}(\mathrm{ob})>,
$$

with $\mathrm{S}_{1}(\mathrm{ob})=\mathrm{R}^{\mathrm{N}}, \mathrm{S}_{2}(\mathrm{ob})=\Re$ - the totality of relations between objects

$$
\mathrm{R}_{\mathrm{n}}(\mathrm{ob}, \mathrm{ob}) \subseteq \mathrm{Ob} \times \mathrm{Ob}, \mathrm{n}=1, \mathrm{~N} .
$$

It's clear that $\sigma^{\mathrm{F}}$ is a representation of $1-(\rightarrow)$ which is the element of Signature of ST(event). So as well as in MetaOnt $^{\text {NAT }}$ each element of Signature of MetaOnt ${ }^{\text {DEDS }}$ has its representation in the elements of Support.

Elementary Operational DEDS $\sigma^{\mathrm{F}}$ is the GRP-solutions for MetaOnt ${ }^{\mathrm{DEDS}}$ that was constructed by extension of structural type $\mathrm{ST}(\mathrm{ob})=\mathrm{M}$ to Evt.

\subsection{Whole representation of Elementary Operational} DEDS. The Elementary Operational DED-System

$$
\left.\boldsymbol{\sigma}^{\mathrm{F}}: \operatorname{evt}_{0}{ }^{\mathrm{evt} 1}[\mid \rightarrow]\right] \mathrm{evt}_{2}
$$

is a complex correspondence that includes three correspondences

- in objects $\mathrm{Ob}$

- in states $\mathrm{S}(\mathrm{ob})$

- in time $\mathrm{T}$

$$
\begin{aligned}
& \mathbf{F}: \mathrm{ob}_{0}{ }^{\mathrm{ob} 1} \rightarrow \mathrm{ob}_{2} \\
& \mathrm{~s}_{0}{ }^{s 1} \rightarrow \mathrm{s}_{2} \\
& \mathrm{t}_{0}^{\tau(\rightarrow)} \rightarrow \mathrm{t}_{2}
\end{aligned}
$$

The first correspondence determines $\sigma^{\mathrm{F}}$ as the algebraic operation $\mathrm{F}$ in $\mathrm{Ob}$.

The purpose of this Section is to determine the control condition that indicates the possibility of performance of operation $\mathbf{F}$ in $\mathrm{Ob}$ on the base of logical form of language $£\left(\right.$ MetaOnt $\left.^{\text {Evt }}\right)$.

Let the elementary event assertion

[evt $]=[$ object ob is in the state $\mathrm{s}$ at a time element $\mathrm{t}]$ corresponds to "physical point" (ob, s, t).

Let the elementary operational assertion

$$
\left[\mathrm{E}\left(\mathrm{ob}_{1}, \mathrm{~F}, \mathrm{ob}_{0}\right)\right]=
$$$$
=\left[\text { object } o b_{1} \text { applies operation } f \text { to object } o b_{0}\right]
$$

corresponds to the arrow $\mid \rightarrow$ from $\sigma^{\mathrm{F}}$.

Then in logical form of $£\left(\mathrm{MetaOnt}^{\text {Evt }}\right)$ the functioning of system $\sigma^{\mathrm{F}}$ in event-space Evt may be given by the conditional assertion $\left[\sigma^{\mathrm{F}}\right]$

$$
\left(\left[\mathrm{evt}_{0}\right] \vee\left[\mathrm{evt}_{1}\right]\right) \wedge\left[\mathrm{E}\left(\mathrm{ob}_{1}, \mathrm{~F}, \mathrm{ob}_{0}\right)\right] \Rightarrow\left[\mathrm{evt}_{2}\right]
$$

Let's denote the condition that indicates the possibility of performance of operation $\mathrm{F}$ in $\mathrm{Ob}$ by

$$
\mathrm{U}^{*}=\mathrm{U}^{*}\left(\mathrm{evt}_{0}, \mathrm{evt}_{1}\right) .
$$

The general model of $\boldsymbol{\sigma}^{\mathrm{F}}$ in Evt is the ordered set of events

$$
\left.\operatorname{Ord}\left(\boldsymbol{\sigma}^{\mathrm{F}}\right)=<\operatorname{Evt}\left(\boldsymbol{\sigma}^{\mathrm{F}}\right) ; \leq\right\}>,
$$

where

$$
\operatorname{Evt}\left(\boldsymbol{\sigma}^{\mathrm{F}}\right)=\left\{\mathrm{evt}_{0}, \mathrm{evt}_{1}, \mathrm{E}\left(\mathrm{ob}_{1}, \mathrm{~F}, \mathrm{ob}_{0}\right)\right\} .
$$

The order $\leq$ may be determined in the form of Petri Net of $\sigma^{\mathrm{F}}$ constructed from follows elementary Petri Nets of logical forms of elements of $\operatorname{Evt}\left(\boldsymbol{\sigma}^{\mathrm{F}}\right)$ :

a) for each elementary event assertion $\left[\mathrm{evt}_{\mathrm{k}}\right], \mathrm{k}=0,1,2$ the correspondences

$$
\begin{gathered}
{\left[\mathrm{ob}_{\mathrm{k}}\right] \Rightarrow\left[\mathrm{ob}_{\mathrm{k}}\right]-\text { place }} \\
\left.\mathrm{t}_{\mathrm{k}} \Rightarrow\right|^{\mathrm{t} \mathrm{k}}-\text { transition } \\
\mathrm{s}_{\mathrm{k}} \Rightarrow \bullet_{\mathrm{k}}-\text { mark }
\end{gathered}
$$

give the Petri model of elementary event assertion [evt $\mathrm{k}_{\mathrm{k}}$

$$
\left.\left(\left(\left[\mathrm{ob}_{\mathrm{k}}\right], \mathrm{s}_{\mathrm{k}}\right) \mathrm{t}_{\mathrm{k}}\right) \Rightarrow\left(\left[\mathrm{ob}_{\mathrm{k}}\right] \mathrm{s}_{\mathrm{k}}\right) \rightarrow\right|^{\mathrm{tk}}
$$

b) for each elementary operational assertion $\left[\mathrm{E}\left(\mathrm{ob}_{1}, \mathrm{~F}, \mathrm{ob}_{0}\right)\right]$ the correspondences

$$
\begin{gathered}
{\left[\mathrm{E}\left(\mathrm{ob}_{1}, \mathrm{~F}, \mathrm{ob}_{0}\right)\right] \Rightarrow[\mathrm{E}]-\text { place }} \\
\left.\mathrm{t}^{*} \Rightarrow\right|^{\mathrm{t} *}-\text { transition } \\
\tau(\rightarrow) \Rightarrow \bullet-\text { mark }
\end{gathered}
$$

where $\mathrm{t}^{*}=\max \left(\mathrm{t}_{0}, \mathrm{t}_{1}\right)+\tau(\rightarrow)$

give the Petri model of elementary operational assertion is

$$
\left(\left.\left(\mathrm{E}\left(\mathrm{ob}_{1}, \mathrm{~F}, \mathrm{ob}_{0}\right), \mathrm{t}^{*}\right) \Rightarrow[\mathrm{E}] \rightarrow\right|^{\mathrm{t}} .\right.
$$

Then Petri Net of $\sigma^{\mathrm{F}}$ which corresponds to ordered set $\operatorname{Ord}\left(\sigma^{\mathrm{F}}\right)$ has the following form

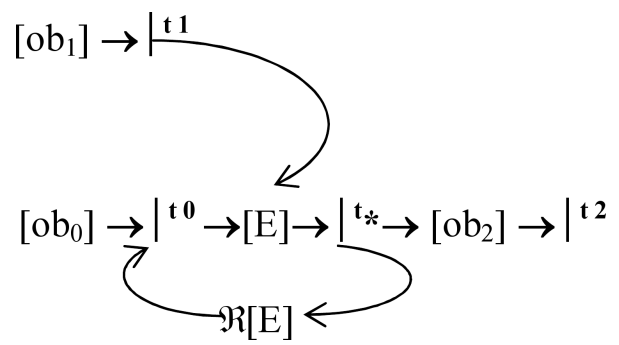

where

$$
\begin{aligned}
& {[E]=} {\left[\mathrm{E}\left(\mathrm{ob}_{1}, \mathrm{~F}, \mathrm{ob}_{0}\right)\right], } \\
& \Re[\mathrm{E}]=\Re\left[\mathrm{E}\left(\mathrm{ob}_{1}, \mathrm{~F}, \mathrm{ob}_{0}\right)\right], \\
& \mathrm{t}_{2} \geq \mathrm{t}^{*} .
\end{aligned}
$$

This Petri Net shows that the possibility to start the performance of operation $\mathbf{F}$ at a time moment $\mathrm{t}_{0} \in \mathrm{T}$ is determined by verity of the assertion

$$
\left(\left[\mathrm{evt}_{0}\right] \vee\left[\mathrm{evt}_{1}\right]\right) \wedge \Re\left[\mathrm{E}\left(\mathrm{ob}_{1}, \mathrm{~F}, \mathrm{ob}_{0}\right)\right] .
$$


So, it is exactly the condition $\mathrm{U}^{*}(9)$ and if $\mathrm{U}^{*}$ is true then transitions $\left.\right|^{\text {t } 0}$ and $\left.\right|^{\text {t1 }}$ must be opened.

The verity of condition $\mathrm{U}^{*}$ depends on the elements of dynamics $\left(\mathrm{s}_{0}, \mathrm{t}_{0}\right)$ and $\left(\mathrm{s}_{1}, \mathrm{t}_{1}\right)$. Thus verity of condition $\mathrm{U}^{*}$ needs any coordination condition

$$
\text { Cord }\left(\mathrm{s}_{0}\left(\mathrm{t}_{0}\right), \mathrm{s}_{1}\left(\mathrm{t}_{1}\right)\right) \text { or }<\operatorname{Cord}\left(\mathrm{s}_{0}, \mathrm{~s}_{1}\right) \text {, Cord }\left(\mathrm{t}_{0}, \mathrm{t}_{1}\right)>
$$

that controls the dependency between the elements of dynamics.

Let Cord $\left(\mathrm{s}_{0}, \mathrm{~s}_{1}\right)$ is true. For example, if $\left\{<\mathrm{ob}_{0}(\mathrm{k})\right.$, $\left.\mathrm{ob}_{1}(\mathrm{k})>\right\}, \mathrm{k} \in \mathbf{N}$ is a sequence of attributions

$$
\mathrm{dom} \Rightarrow \mathrm{ob}_{0}(\mathrm{k}) \text { and cen } \Rightarrow \mathrm{ob}_{1}(\mathrm{k})
$$

then Cord $\left(t_{0}, t_{1}\right)$ is represented in the form of dynamics of starting time moments $\mathrm{t}_{0}$ that is given by the $(\max ,+)$ - equation

$$
\mathrm{t}_{0}(\mathrm{k}+1)=\mathrm{t}_{0}(\mathrm{k}) \otimes \mathrm{t}_{1}(\mathrm{k}) \oplus \tau(\rightarrow)
$$

So, condition

$$
\text { Cord }\left(t_{0}, t_{1}\right)
$$

is responsible for coordination in time totality $\mathrm{T}$ (synchronization) and condition

$$
\text { Cord }\left(\mathrm{s}_{0}, \mathrm{~s}_{1}\right)
$$

is responsible for the coordination in state space $\mathrm{S}(\mathrm{ob})$.

5.3. Complex Operational DEDS. Let $\sigma_{\mathrm{k}}^{\mathrm{F}}, \mathrm{k}=1, \mathrm{~N}$ be the Elementary Operational DEDS. Let call the events evt $\mathrm{k}_{0}, \mathrm{evt}_{\mathrm{k} 1}$ incoming and the events evt $t_{\mathrm{k} 2}$ outgoing for each of $\sigma_{\mathrm{k}}^{\mathrm{F}}$. If the outgoing events of $\sigma_{\mathrm{k}}^{\mathrm{F}}$ are the incoming for $\sigma_{\mathrm{m}}^{\mathrm{F}}$ then these types of events induce the technological link

$$
\mathrm{R}_{\mathrm{TH}}=\mathrm{R}_{\mathrm{TH}}\left(\sigma_{\mathrm{k}}^{\mathrm{F}}, \sigma_{\mathrm{m}}^{\mathrm{F}}\right)
$$

on totality $\Sigma(7)$. Thus the following structure

$$
\Sigma=<\left\{\sigma_{\mathrm{k}}^{\mathrm{F}} \mid \sigma_{\mathrm{k}}^{\mathrm{F}}, \mathrm{k}=1, \mathrm{n} .\right\}, \mathrm{R}_{\mathrm{TH}}>
$$

is the Complex Operational DEDS and it may be considered as a the system model of "technology".

\subsection{Complex Temporal DEDS or system model of object} totality. The metaontology MetaOnt ${ }^{\text {Evt }}$ allows one interesting model of totality of objects in Ob that cannot be discovered in metaontology MetaOnt ${ }^{\mathrm{NAT}}$.

The systems

$$
\begin{gathered}
\sigma_{1}^{\mathrm{T}}=<\mathrm{ob}_{1}, \partial\left(\mathrm{ob}_{1}\right)> \\
\sigma_{2}^{\mathrm{T}}=<\mathrm{ob}_{2}, \partial\left(\mathrm{ob}_{2}\right)>,
\end{gathered}
$$

are Elementary Temporal DEDS where $\mathrm{ob}_{1}, \mathrm{ob}_{2}$ are any elements of totality of objects $\mathrm{V} \subseteq \mathrm{Ob}$.

The following relation between elements of dynamics $\partial\left(\mathrm{ob}_{1}\right)$ and $\partial\left(\mathrm{ob}_{2}\right)$ from $\sigma_{1}^{\mathrm{T}}$ and $\sigma_{2}^{\mathrm{T}}$ that is initially given as symbolic conditional assertion in $£\left(\mathrm{MetaOnt}^{\mathrm{Evt}}\right)$ :

$$
\begin{gathered}
\mathrm{L}\left(\rightarrow_{\mathrm{N}}\right)=\exists\left(\mathrm{s}_{1}, \mathrm{t}_{1}\right) \exists\left(\mathrm{s}_{1}^{\prime}, \mathrm{t}_{1}^{\prime}\right)\left[\left(\mathrm{ob}_{1},\left(\mathrm{~s}_{1}, \mathrm{t}_{1}\right)\right) \rightarrow\right. \\
\left.\rightarrow\left(\mathrm{ob}_{1},\left(\mathrm{~s}_{2}, \mathrm{t}_{2}\right)\right)\right] \rightarrow_{\mathrm{N}}\left[\left(\mathrm{ob}_{2},\left(\mathrm{~s}_{1}^{\prime}, \mathrm{t}_{1}^{\prime}\right)\right) \rightarrow\left(\mathrm{ob}_{2},\left(\mathrm{~s}_{2}^{\prime}, \mathrm{t}_{2}^{\prime}\right)\right)\right]
\end{gathered}
$$

and presented in the form of the diagram

$$
\begin{aligned}
\left(\mathrm{ob}_{1},\left(\mathbf{s}_{\mathbf{1}}, \mathbf{t}_{\mathbf{1}}\right)\right) & \underset{\downarrow_{\mathrm{p}}}{\rightarrow}\left(\mathrm{ob}_{1},\left(\mathrm{~s}_{2}, \mathrm{t}_{2}\right)\right) \\
& \left(\mathrm{ob}_{2},\left(\mathbf{s}_{\mathbf{1}}^{\prime}, \mathbf{t}_{\mathbf{1}}^{\prime}\right)\right) \rightarrow_{\mathrm{q}}\left(\mathrm{ob}_{2},\left(\mathrm{~s}_{2}^{\prime}, \mathrm{t}_{2} ;\right)\right)
\end{aligned}
$$

is called the system link between the objects $\mathrm{ob}_{1}$ and $\mathrm{ob}_{2}$ [8].

The system link is a correspondence between the elements of dynamics of objects $o b_{1}$ and $o b_{2}$

$$
\begin{aligned}
& \partial\left(\mathrm{ob}_{1}\right): \mathrm{T} \rightarrow \mathrm{S}\left(\mathrm{ob}_{1}\right), \\
& \partial\left(\mathrm{ob}_{2}\right): \mathrm{T} \rightarrow \mathrm{S}\left(\mathrm{ob}_{2}\right),
\end{aligned}
$$

and it may be given by the following mapping

$$
\lambda: \mathrm{S}\left(\mathrm{ob}_{1}\right) \times \mathrm{T} \rightarrow \mathrm{N} \mathrm{S}\left(\mathrm{ob}_{2}\right) \times \mathrm{T},
$$

supplemented with a certain condition

$$
\text { Cord }\left(\mathrm{s}_{0}\left(\mathrm{t}_{0}\right), \mathrm{s}_{1}\left(\mathrm{t}_{1}\right)\right)
$$

or

$$
<\operatorname{Cord}\left(\mathrm{s}_{0}, \mathrm{~s}_{1}\right) \text {, Cord }\left(\mathrm{t}_{0}, \mathrm{t}_{1}\right)>
$$

responsible for $\partial\left(\mathrm{ob}_{1}\right)$ and $\partial\left(\mathrm{ob}_{2}\right)$ coordination.

Thus system link $\rightarrow_{\mathrm{N}}\left(\mathrm{ob}_{1}, \mathrm{ob}_{2}\right)$ is related to the elements of dynamics but at the same time it explains the dependency between objects in totality $\mathrm{V} \subseteq \mathrm{Ob}$ as a relation "object $\mathrm{ob}_{1}$ depends on object $\mathrm{ob}_{2}$ ".

In accordance with diagram (10) the sense of dependency $\rightarrow_{\mathrm{N}}\left(\mathrm{ob}_{1}, \mathrm{ob}_{2}\right)$ may be represented by conceptual assertion from $£\left(\right.$ MetaOnt $\left.^{\text {Evt }}\right)$

$£(\rightarrow \mathrm{N})=$ "if object ob $_{1}$ changes its state $\mathbf{s}_{\mathbf{1}}$ at time moment $\mathbf{t}_{1}$ then the object ob $_{2}$ changes its state $\mathbf{s}_{1}^{\prime}$, at time moment $\mathbf{t}_{1}^{\prime}$ ", that corresponds to conditional assertion $\mathrm{L}\left(\rightarrow_{\mathrm{N}}\right)$.

So, totality of objects $\mathrm{V} \subseteq$ Ob becomes Complex Temporal DEDS which has the following mathematical model

$$
\Sigma=<\text { Evt, } \rightarrow \mathrm{N}(\mathrm{x}, \mathrm{y})>\text {. }
$$

Let $\mathrm{ob}_{1}, \mathrm{ob}_{2} \in \mathrm{V}$ be two any elementary conceptual objects

$$
\begin{gathered}
\mathrm{ob}_{1}=<\left[\mathrm{ob}_{1}\right], \mathrm{A}\left(\mathrm{ob}_{1}\right)>, \\
\mathrm{ob}_{2}=<\left[\mathrm{ob}_{2}\right], \mathrm{A}\left(\mathrm{ob}_{2}\right)>
\end{gathered}
$$

and $\mathrm{S}(\mathrm{A}(\mathrm{ob}))$ is the state space of elementary class $\mathrm{A}(\mathrm{ob})$.

Then $\rightarrow_{\mathrm{N}}\left(\mathrm{ob}_{1}, \mathrm{ob}_{2}\right)$ has two forms:

$$
\begin{gathered}
{\left[\mathrm{ob}_{1}\right] \rightarrow_{\mathrm{N}}\left[\mathrm{ob}_{2}\right]-\text { graph ark }} \\
\lambda: \mathrm{S}\left(\mathrm{A}\left(\mathrm{ob}_{1}\right)\right) \times \mathrm{T} \rightarrow \mathrm{N} \mathrm{S}\left(\mathrm{A}\left(\mathrm{ob}_{2}\right)\right) \times \mathrm{T} .
\end{gathered}
$$

The first form presents the complex Temporal DEDsystem $\Sigma$ in the form of any graph $G$ that can be marked. The functions $\lambda$ associated with graph's arks make $G$ functional [9].

The correspondence (Subsec. 5.2)

$$
\left.(([\mathrm{ob}], \mathrm{s}), \mathrm{t}) \Rightarrow([\mathrm{ob}], \mathrm{s}) \rightarrow\right|^{\mathrm{t}},
$$

transforms this graph in the form of Petri Net. If $\mathrm{S}(\mathrm{A}(\mathrm{ob}))=$ $\mathrm{R}^{\mathrm{N}}$ then this Petri Net becomes continuos and as it was shown in [1] the dynamics of complex Temporal DEDS (11) takes the form of system differential equations. 


\section{Discussion}

One of the most important problems in the theory of software engineering is the problem of encapsulation which. consists to separate the modeling level of user from programming level.

The modern theory of information systems says that the solution of this problem takes the form of universal or unified modeling language. If any unified modeling language is the language of any structural type then DEDS models from Sec. 5 presents one of the possible solutions of encapsulation problem.

The first solution - Complex Operational DEDS was constructed in the form of system model of the algebraic operation. In comparison with Elementary Functional SADTmodel the Elementary Operational DEDS allows to supplement the "input-output" (i.e. object-oriented) functional model with control condition in event space, i.e. space of the states of objects .

The second solution - Complex Temporal DEDS gives the system understanding of a totality of objects. Namely, a totality of objects is the system if there exist the system link or dependency between the states of the objects that compose it. For example, V(lakes) - the totality of lakes from [11] is the system because there is the system link $£\left(\rightarrow_{N}\right)=$ "level of water in the lake x s from level of water in the lake y" where level of water is the numerical character-state of the object lake. The dynamics of totality $\mathrm{V}$ (lakes) takes the form of the system of difference equations.

\section{Conclusions}

The construction of model of discrete event dynamic system (DEDS) was considered. The modeling process was realized on the base of Ontological approach. Ontological approach joins the conceptual and mathematical levels of modeling process and consists in follow steps:

- to construct the metaontology (conceptual modeling level);

- to construct the GRP-solutions (structural types) for this metaontology (mathematical modeling level).

There was constructed metaontology of physical point and there were constructed two its GRP-solutions. The first solution is Complex Operational DEDS

$$
\mathrm{COD}(\mathrm{evt})=<\text { Evt, } \mathrm{A}(\rightarrow)>
$$

which is conceptually common to the differential system models. The Petri model of Elementary Operational DEDS was studied.

The second solutions is Complex Temporal DEDS

$$
\left.\mathrm{CTD}(\mathrm{evt})=<\mathrm{Evt}, \rightarrow_{\mathrm{N}}(\mathrm{x}, \mathrm{y})\right\rangle
$$

which takes the form of dynamics of totality of objects provided with the system link (dependency) $\rightarrow_{\mathrm{N}}(\mathrm{x}, \mathrm{y})$. It was noted that Complex Temporal DEDS has representation in the form of continuous Petri Net.

\section{REFERENCES}

[1] R. Kara, S. Djennoune, and J-J.Loiseau, "State feedback control for manufacturing systems modeled by continuos Petri Nets", $12^{\text {th }}$ IFAC Symposium on Control Problems in Manufacturing 1, 379-384 (2006).

[2] S.N. Nikolsky, "Ontological models of information control systems life cycle", RF Conf. with Int. Participation 2, 184-190 (2007), (in Russian).

[3] K.E. Wolf, "Applications of temporal conceptual semantic system", RF Conf. with Int. Participation 1, 3-15 (2007).

[4] N. Guarino, "Formal ontology and information systems", Proc. FOIS 6-8, 3-15 (1998).

[5] C.V. Negoita, Management Applications of System Theory, Verlag, Basel-Stuttgart, 1979.

[6] I.A. Lomazova, "Nested petri nets - a formalism for specification and verification of multi-agent disributed systems", Fundamenta Informaticae 43 (1-4), 195-214 (2000).

[7] R. Goldblatt, TOPOI. The Categorial Analysis of Logic, Oxford University Press, Oxford, 1979.

[8] S.N. Nikolsky, "The pecularities of purposeful behaviour formalization. System analysis", Modeling, Simulation 3 (4), 359364 (1986).

[9] V.V. Kulba, D.A. Kononov, C.A. Kosiachenko, and A.N. Shoobin, "Methods of forming of scenarios of social economic system development", M. SINTEG, CD-ROM (2004), (in Russian).

[10] F.S. Roberts, Discrete Mathematical Models with Applications to Social, Biological and Enviromental Problems, PrenticeHall, New Jersey, 1976.

[11] J. Casti, Connectivity, Complexity, and Catastrophe in Largescale Systems, John Wiley\&Sons, Oxford, 1979. 